\title{
Imbalanced Distribution of Plasmodium falciparum MSP-1 Genotypes Related to Sickle-Cell Trait
}

\author{
Francine Ntoumi, ${ }^{1,4}$ Christophe Rogier, ${ }^{2}$ Alioune Dieye, \\ Jean-François Trape, ${ }^{3}$ Pascal Millet, ${ }^{4}$ and \\ Odile Mercereau-Puijalon ${ }^{1}$ \\ ${ }^{1}$ Unité d'Imunologie Moléculaire des Parasites, Institut Pasteur, Paris, \\ France ${ }^{2}$ Institut Pasteur, Dakar, Senegal ${ }^{3}$ Laboratoire de \\ Paludologie, ORSTOM, Dakar, Senegal ${ }^{4}$ International Centre for \\ Medical Research, Franceville, Gabon
}

\begin{abstract}
Background: The sickle-cell trait protects against severe Plasmodium falciparum malaria and reduces susceptibility to mild malaria but does not prevent infection. The exact mechanism of this protection remains unclear. We have hypothesized that AS individuals are protected by virtue of being less susceptible to a subset of parasite strains; thus we compared some genetic characteristics of parasites infecting AS and AA subjects.

Materials and Methods: Blood was collected from asymptomatic individuals living in two different regions of Africa. The polymorphic MSP-1 and MSP-2 loci were genotyped using a PCR-based methodology. Individual alleles were identified by size polymorphism, amplification using family-specific primers, and hybridization using family-specific probes. Multivariate logistic regression was used to analyze allele distribution.
\end{abstract}

Results: In Senegalese carriers, age and hemoglobin type influenced differently the distribution of the three MSP-1 families and had an impact on distinct individual alleles, whereas the distribution of MSP-2 alleles was marginally affected. There was no influence of other genetic traits, including the HLA Bw53 genotype, or factors such as place of residence within the village. In a cohort of Gabonese schoolchildren in which the influence of age was abrogated, a similar imbalance in the MSP-1 allelic distribution but not of MSP-2 allelic distribution by hemoglobin type was observed.

Conclusions: The influence of the host's hemoglobin type on $P$. falciparum genotypes suggests that parasite fitness for a specific host is strain-dependent, which is consistent with our hypothesis that innate resistance might result from reduced fitness of some parasite strains for individuals with sickle-cell traits.

\section{INTRODUCTION}

Several genetic traits protect against life-threatening Plasmodium falciparum malaria. In subSaharan Africa where $P$. falciparum malaria is a

Christophe Rogier's present address is Laboratoire de Parasitologie, Institut de Médecine Tropicale du Service de Santé des Armées, Marseilles, France.

Address correspondence and reprint requests to: Dr. Odile Mercereau-Puijalon, Unité d'Imunologie Moléculaire des Parasites, Institut Pasteur, 25 rue du Dr Roux, 75724 Paris, France. Phone: 3301456886 23; Fax: 33014061 31 85; e-mail: omp@pasteur.fr major cause of childhood mortality, the bestdocumented innate protective factor is the sickle-cell trait $(1,2)$. There is convincing evidence that AS subjects are protected against severe forms of malaria (3-5) and have a reduced susceptibility to mild malaria with regard to their AA and SS counterparts $(3,4,6)$. The mechanism to which AS subjects owe this protection is still poorly understood. It has been suggested that the selective advantage of the sickle-cell trait might reside in rapid acquisition of immunity $(7,8)$. In vitro studies have shown that parasite develop- 
ment in AS red blood cells was impaired under low oxygen tension $(9,10)$ and that ring-stage infected AS cells had a significantly higher sickling rate than noninfected cells (11). Such effects can only be observed at an oxygen tension unlikely to occur in vivo (12). More importantly, these in vitro data implying that protection is due to markedly reduced parasite proliferation do not explain why AS individuals still remain susceptible to uncomplicated forms of malaria that are associated in endemic areas with substantial peripheral parasite densities (13) and why parasite prevalence and densities are usually similar in asymptomatic AS and AA subjects $(6,14)$.

In 1954, Allison experimentally inoculated Kenyan adults who had left an endemic area for 18 months with $P$. falciparum parasites and observed that AS volunteers were less readily infected than AA individuals. He concluded that sickle-cell trait carriers had a reduced susceptibility to infection (15). As this is in apparent contradiction with similar parasite rates in AS and AA subjects, we formulated the hypothesis that the host's hemoglobin type influences infectiousness of certain parasite strains, resulting in AS individuals being less likely to host a subset of parasites. The corollary of this, namely that parasite strains differ in their infectiousness for a particular host, is indicated by our recent observation that different $P$. falciparum strains varied markedly in their capacity to infect nonimmune, laboratory-bred Saimiri sciureus monkeys with similar genetic background (16).

To investigate the hypothesis that sickle-cell trait influences the type of parasite strains able to successfully establish a blood stage infection, we have compared some molecular characteristics of $P$. falciparum parasites infecting AS and AA individuals. $P$. falciparum genotyping was carried out using two polymorphic markers, MSP-1 and MSP-2. These single copy genes code for major surface antigens of the merozoite, the extracellular stage invading the erythrocyte. The exact biological function of both proteins is still unclear. MSP-1 is produced during the second half of the intraerythrocytic cycle as a high molecular mass protein, which undergoes sequential proteolytic processing at the end of schizogony (17). MSP-2 is produced late in the cycle and, like MSP-1, is exposed on the surface of the merozoite (18). Both genes have a very large number of alleles, grouped into three and two allelic families, respectively. Within an allelic family, alleles have a high degree of homology, and they differ by point mutations and the number and arrange- ment of repeated sequences. Between allelic families, there is considerable sequence divergence, resulting in the absence of cross-hybridization of the polymorphic domains at the nucleic acid level and absence of immunologic cross-reactivity $(18,19)$. Thanks to their large polymorphism, these markers constitute remarkably powerful tools for parasite genotyping, enabling us to rapidly characterize field isolates and determine the number of distinct clones present in mixed infections $(20-25)$. They have been used here to analyze isolates collected from $P$. falciparum infected inhabitants of two villages located in different endemic areas of Africa-Senegal (West Africa) and Gabon (Central Africa).

\section{MATERIALS AND METHODS}

\section{Donors and Genetic Factors}

Blood was collected in Dielmo, a holoendemic village of Senegal, from 22 July to 6 August 1992, when the entomological inoculation rate averaged 1 infective bite/person/night (26). In order to study natural host-parasite interactions with minimal interference from external factors, we recruited asymptomatic parasite carriers who had not left the village for a minimum of 1 month before blood collection and who had not received any anti-malarial medication during the preceding 3 weeks or more. Ages ranged from 1 to 84 years. There were 39 male and 38 females. The villagers recruited were from 21 closely located compounds situated within a $24,000 \mathrm{~m}^{2}$ triangle. The compounds were all located within 30-150 m from main mosquito breeding sites. The 20 AS individuals lived in 10 distinct compounds scattered throughout the village. For each AS individual there was at least one AA person living in the same compound. Informed consent was obtained from donors or the parents before collection of blood samples. A large number of parameters potentially influencing hostparasite relationships were available for each inhabitant of the village, including $\mathrm{ABO}$ and Rhesus blood groups, Glucose-6-phosphate dehydrogenase levels, time of residence in the village and travels away from Dielmo, usage of bednets, individual history of clinical episodes, and parasitologic follow-up. Experimental procedures for obtaining this body of data have been described elsewhere (26).

A cohort of 163 asymptomatic schoolchildren, aged 7-14 years, was recruited in the Di- 
enga village, Gabon (27) during the 1995 rainy season. Children who did not present malaria symptoms the 2 weeks preceding and the day after the blood sampling were included in this work. The Saker-Solomon test was used to detect any antimalarial medication in urine. Recruitment was made during several visits to school as follows: $27 \mathrm{AA}$ and 9 AS on 2/21/95; $13 \mathrm{AA}$ and 5 AS on $3 / 6 / 95 ; 11$ AA and 4 AS on $3 / 21 / 95 ; 7$ $\mathrm{AA}$ and 3 AS on $3 / 22 / 95 ; 27 \mathrm{AA}$ and 7 AS on 4/4/95; 22 AA and 7 AS on 4/25/95; 16 AA and 5 AS on $5 / 16 / 95$. There were 87 boys and 76 girls, equally distributed in each sampling day. The mean age group was 10.36 years. The children were from 62 different homes located in different parts of the village. Venous blood samples were collected on EDTA. Informed consent was obtained from the parents.

Thick blood smears were examined and parasite density quantified as described (26). Hemoglobin type was determined by standard cellulose acetate electrophoresis, and HLA typing was carried out as reported (28).

\section{DNA Extraction and Polymerase Chain Reaction}

DNA was extracted from frozen red blood cell pellets as described earlier (23). Genotyping of the MSP-1 and MSP-2 loci was done by analysis of the polymorphic MSP-1 block 2 and of the central polymorphic MSP-2 domain. For the MSP-1 gene, the products of the primary polymerase chain reaction (PCR) made using primers A +B (23) were separated by agarose gel electrophoresis and assigned to one of the three major allelic families by hybridization using familyspecific probes $(23,25)$. This was confirmed by a nested PCR driven by one of the three homologous pairs of family-specific primers (25). For the MSP-2 locus, alleles were amplified by nested PCR driven, using primers derived from the conserved $5^{\prime}$ and $3^{\prime}$ regions, namely, primers $1+4$ and $2+3$, for the first and second reaction, respectively. Products were separated by agarose gel electrophoresis and assigned to one of the three major allelic families by hybridization using family-specific probes under stepwise increasing stringency as described elsewhere $(24,25)$. Bands generating a strong signal at high stringency $\left(0.1 \times \mathrm{SSC}\right.$ at $\left.65^{\circ} \mathrm{C}\right)$ with one probe were considered as alleles belonging to that family. Fragments giving strong hybridization signals under conditions of moderate stringency $(0.5 \times$ $\mathrm{SSC}, 65^{\circ} \mathrm{C}$ ) but failing to hybridize at high strin- gency $\left(0.1 \times \mathrm{SSC}, 65^{\circ} \mathrm{C}\right)$ were considered hybrid alleles (intra-genic recombinants) $(24,25)[1 \times$ $\mathrm{SSC}$ is $0.15 \mathrm{M} \mathrm{NaCl}, 0.015 \mathrm{M} \mathrm{Na}_{3}$ citrate, $\mathrm{pH} 7.0$ ]. This assignment was confirmed by nested PCR driven in the second reaction by one of the four combinations of family-specific primers, i.e., using forward and reverse primers derived from one family or using a forward primer from one family combined with a reverse primer from the other family $(24,25)$.

\section{Statistical Analysis}

For the Dielmo cohort, comparison of the complexity (average number of distinct alleles per PCR-positive isolate) in the various groups considered was done using a Mann-Whitney $U$ test. Frequency of the MSP-1 and MSP-2 allelic families was calculated as number of bands assigned to one family divided by total number of bands detected for that locus in one group. Comparison of frequency in the various groups considered was done using a Khi 2 test.

In the analysis of the distribution of individual alleles, allelic frequency within a parasite population was calculated as number of isolates containing a particular allele divided by total number of bands detected for that locus in one particular group. Global comparisons were carried out using the approach of O'Quigley and Schwartz (29), which may be used in the comparison of several proportions in the presence of low cell expectations. This test yielded conclusions similar to those obtained using the test described by Fleiss (30). The O'Quigley and Schwartz test was retained because it was more conservative and had fewer restrictive validity conditions than the Fleiss test. Because the population of interest was the parasite population, the statistical units were the alleles and the variables studied were the nature of the allele (outcome variable: present or absent) and the characters of the carriers (independent variables: age, hemoglobin type, HLA phenotype). When the distribution of alleles was significantly imbalanced and to take into account the possible interrelation of the alleles present simultaneously in the same blood sample as a result of mixed infection, the effects of hemoglobin type and age of carrier were analyzed by a random-effect logistic regression model $(13,31)$. The effect of age was taken into account as a dichotomous variable ( $\leq 10$ years and $>10$ years), because this approach led to the best minimization of the deviance and because the incidence of clinical 
malaria is consistently low above 10 years of age in Dielmo (32). For the alleles showing a mutually exclusive distribution in AA and AS subjects or in subjects $\leq 10$ years and $>10$ years of age, the logistic regression model was unable to fit the data and an exact Fisher test was used. The significance level was calculated using the correction of Fisher (33), namely $5 / n$ percent, where $n$ is the number of comparisons. For 22 tests carried out simultaneously, the significance level applied to each test was therefore $p=0.05 / 22=$ 0.00227 .

A separate analysis considered the frequency of carriage of each allele (outcome variable) in the human population, which was calculated as number of isolates presenting a specific allele divided by the number of individuals within the group considered. Comparisons of allele frequency by host character was done using the Exact Fisher test.

In Dielmo, the proportion of AS subjects did not significantly differ in the 21 compounds as indicated by the $\mathrm{O}^{\prime}$ Quigley and Schwartz Test, $Z=1.5$ nonsignificant (NS) (29) and the test described by Fleiss $Z=1.45$, NS (30). The proportion of AS subjects did not significantly differ by village area (Khi2 Test Khi2 $=1.2, \mathrm{df}=2$, NS).

For the Dienga cohort, frequency of the MSP-1 and MSP-2 allelic families and of the individual alleles in AS and AA subjects were compared using a Khi 2 test. There was no influence of age in allele distribution. Frequency of the MSP-1 and MSP-2 allelic families and the individual alleles in AS and AA subjects, calculated as above, were compared using a Khi 2 test. The children recruited lived in 62 compounds. For each AS child, there was at least one AA child who lived in the same home. A possible impact of the household and the date of the sampling on the allele distribution was considered. No influence of the date of sampling was observed (Khi 2 test). The very low number of alleles detected per home did not allow home-to-home comparisons; however, there was no obvious evidence for any clustering effect.

\section{RESULTS}

Blood samples were collected from 77 asymptomatic individuals of all age-groups living permanently in Dielmo (Senegal, West Africa), where malaria is holoendemic (26). P. falciparum parasites were detected by microscopy in 50 of
77 subjects. PCR analysis, however, indicated a larger proportion of infections, as 67 and 60 individuals were positive for the MSP-1 - and MSP2 -based reactions, respectively (Table 1 ). The discrepancy of microscopic and PCR detection was mainly due to low parasite densities in adults, which frequently were below the sensitivity threshold of microscopy.

The mean peripheral $P$. falciparum density was influenced by the age but not by the genetic traits investigated (data not shown). Three host factors influencing susceptibility to clinical malaria in holoendemic areas were considered and the group was stratified accordingly: (1) by age: the age of 10 years was used as the cut-off, as above this age the incidence of clinical malaria remains consistently low in Dielmo (32); (2) by AA or AS hemoglobin type; and (3) by carriage of the HLA Bw53 allele, which is reported to reduce the risk of severe malaria in Gambian populations (4) whose HLA allele frequency is similar to that of Dielmo villagers (28). The HLA B locus was successfully typed for 67 of 77 enrolled subjects.

MSP-1- and MSP-2 genotyping was performed by PCR, as indicated in Materials and Methods. Individual alleles were identified by size polymorphism and assigned to a particular allelic family by hybridization and nested PCR using family-specific primers. A very large polymorphism was observed for both loci. Seventeen MSP-1 alleles were observed, with 8, 5, and 4 distinct K1-, MAD20-, and RO33-type alleles, respectively (Table 2 ). Likewise, 22 MSP-2 alleles were detected, 8 from the 3D7 family, 7 from the FC27 family, and 7 hybrid alleles (Table 2).

A very large proportion of isolates contained multiple $P$. falciparum clones. As noted previously (25), the complexity (the average number of distinct MSP-1 or MSP-2 alleles identified per PCRpositive isolate) was reduced by age (MannWhitney U test, 0.008). On the contrary, the innate factors considered did not have any impact on complexity of the infection in this cohort (Table 1).

Comparison of the parasite populations by host character showed that the relative frequency of the three major MSP-1 allelic families was influenced by hemoglobin type $(p=0.010)$ and age $(p<0.001)$, as illustrated in Fig. 1. None of the host factors studied influenced distribution of MSP-2 allelic families.

The distribution of individual alleles within the populations stratified by age, hemoglobin type, and HLA-Bw53 type was examined as de- 


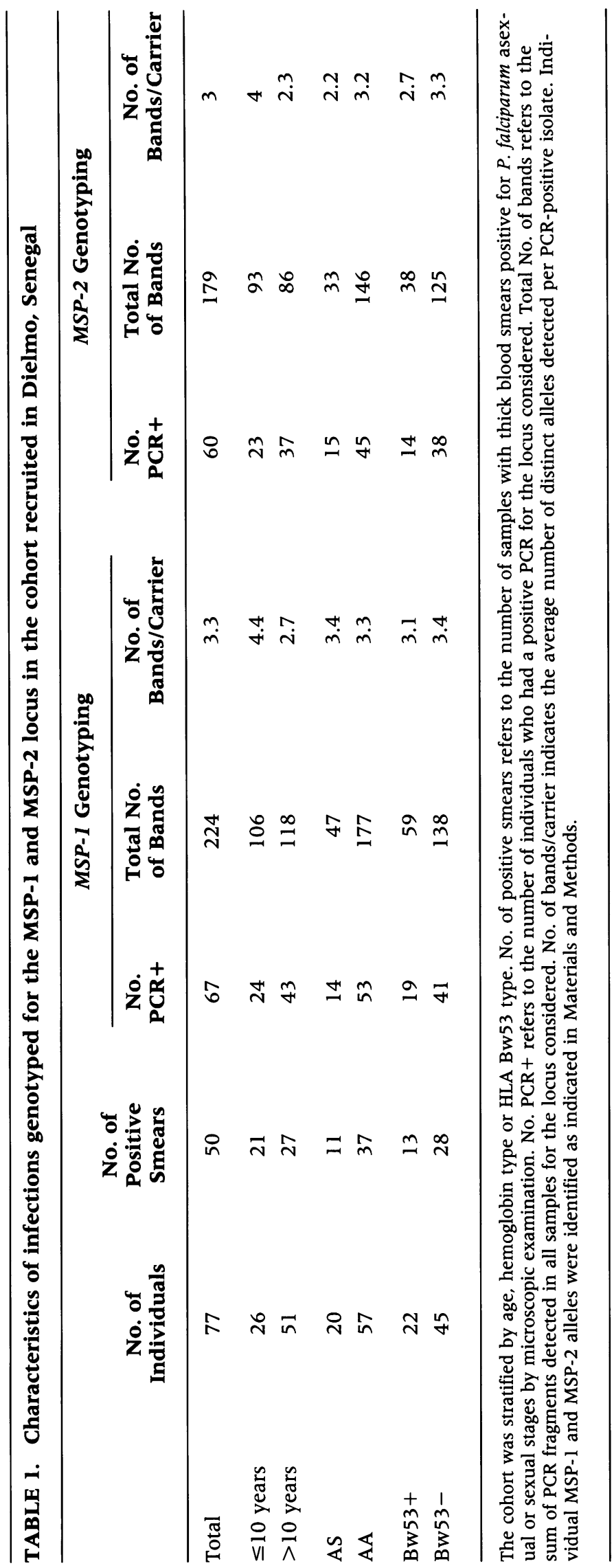




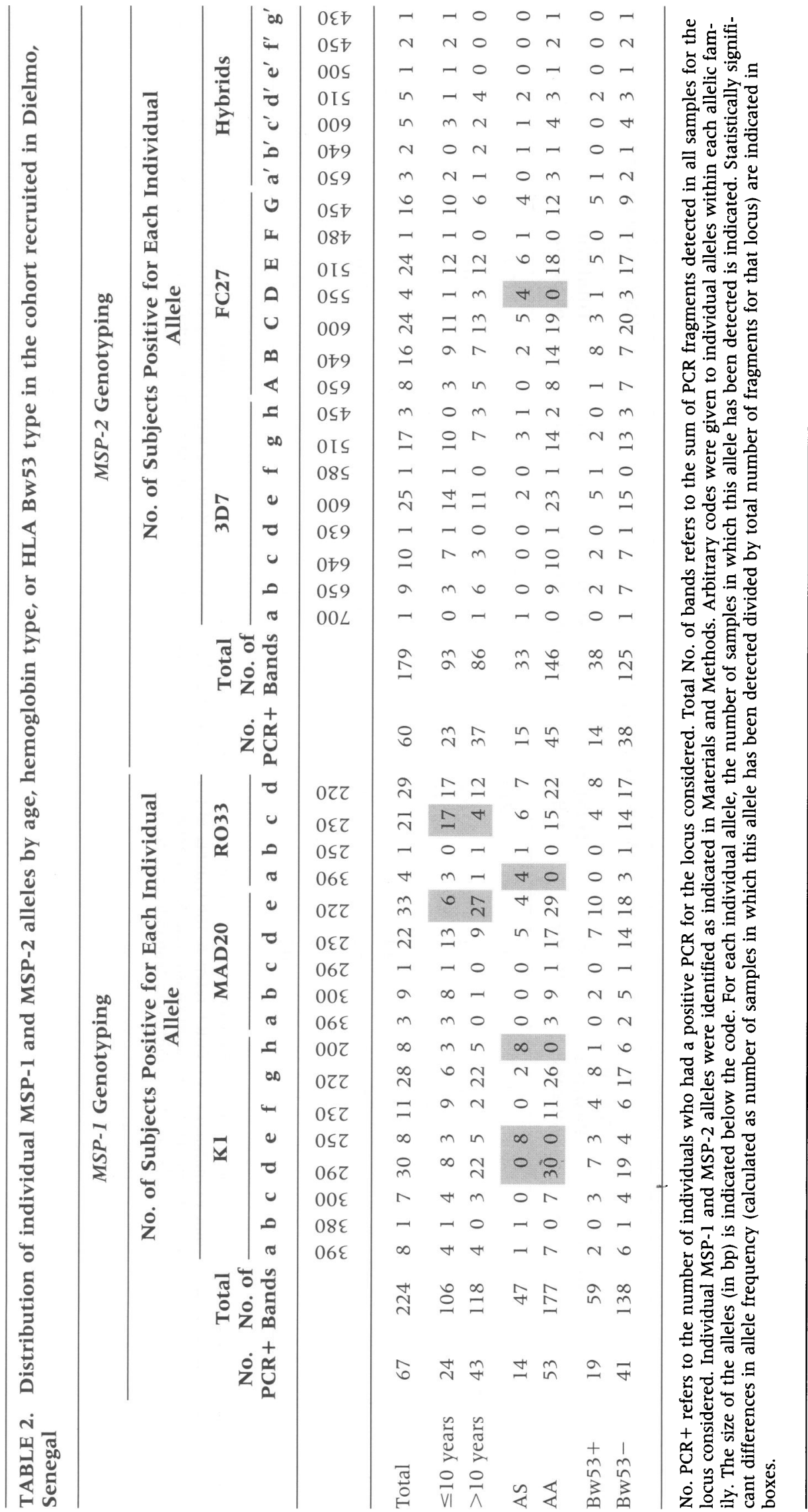




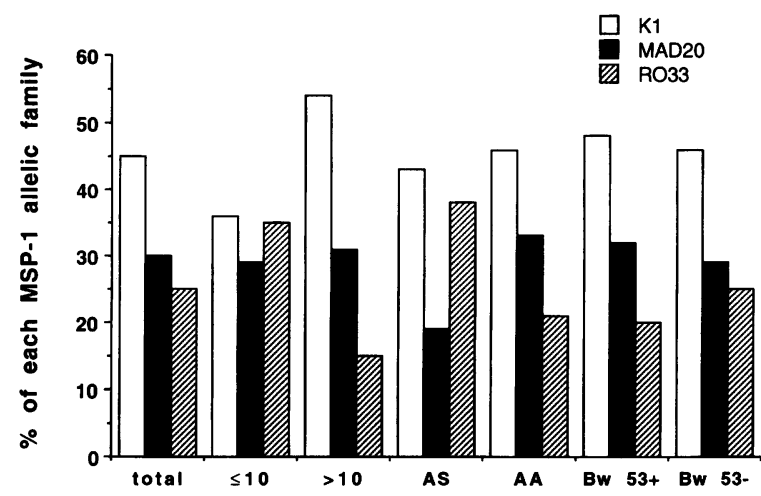

FIG. 1. Frequency of the MSP-1 allelic families in the cohort recruited in Dielmo, Senegal, unstratified (total), and stratified by age, hemoglobin type and presence $(+)$ or absence $(-)$ of the HLA Bw53 allele

Distribution of the various factors investigated was as follows: among the $\leq 10$ year-olds, there were 19 AA (4 Bw53+, 14 Bw53-, and one individual untyped for this locus) and 7 AS children (1 Bw53+, 4 Bw53-, and 2 children untyped for this locus). The AA group ( $19 \leq 10$ years and $38>10$ years) contained $17 \mathrm{Bw} 53+, 33 \mathrm{Bw} 53-$, and 7 individuals untyped for this locus. The AS group $(7 \leq 10$ years and $13>10$ years) contained 5 Bw53+, 12 Bw53-, and 3 subjects untyped for this locus. The Bw53+ group contained $4 \leq 10$ years and $18>10$ years, 17 AA and 5 AS subjects. The group of subjects not harboring the Bw53 allele contained $19 \leq 10$ years and $26>10$ years, 33 AA and 12 AS subjects. Typing of the parasites was done as described in Materials and Methods. Assignment of a PCR fragment to a specific allelic family was done by hybridization using family-specific probes under distinct conditions of stringency and verified by secondary nested PCR using family-specific primers.

scribed in Materials and Methods. Table 2 shows that the frequency of individual MSP-1 alleles was affected by age $\left(\mathrm{O}^{\prime}\right.$ Quigley and Schwartz test $Z=7.8, p<0.000001$; Fleiss test, $Z=7.85, p<$ $0.000001)$ and hemoglobin type $\left(\mathrm{O}^{\prime}\right.$ Quigley and Schwartz test $Z=15.1, p<0.000001$; Fleiss test, $Z=15.7, p<0.000001)$; whereas there was no influence of neither age $\left(\mathrm{O}^{\prime}\right.$ Quigley and Schwartz test, $Z=0.1$, N.S.) nor hemoglobin type $(Z=2.75, p<0.006)$ on the frequency of individual MSP-2 alleles. Interestingly, influence of age and hemoglobin concerned distinct MSP-1 alleles. The MAD20-e allele was significantly more frequent in the population analyzed from $>10$-year-old subjects and conversely, the RO33-c allele was significantly more frequent in the population hosted by the younger age-group (likelihood ratio test, $p=0.0025,<0.001$, and
$<0.001$, respectively). The lower frequency of the K1-g allele in children did not reach significance $(p=0.08)$.

Analysis of the distribution of individual MSP-1 and MSP-2 alleles showing mutually exclusive distribution indicated that the hemoglobin type had a statistically significant influence on the frequency of the K1-e, K1-h, and RO33-a alleles, which was higher in the parasite population infecting AS subjects as compared with that infecting AA subjects (exact Fisher test, $p<$ 0.000001 for Kl-e and $\mathrm{Kl}-\mathrm{h}$ and $p<0.0017$ for RO33-a). Conversely, the frequency of the Kl-d allele was higher in AA than in AS subjects $(p=$ $0.0025)$. The imbalance in the distribution of other alleles was less pronounced and could not be attributed unequivocally to the hemoglobin type of the blood donor. There was no statistically significant influence of the HLA Bw53 genotype on MSP-1 allele frequency (Table 2). The large number of MSP-2 alleles and their dispersed distribution rendered analysis of MSP-2 allele frequency less powerful. The frequency of the FC27-D allele was significantly higher in the parasite population from AS subjects as compared with that from AA subjects (exact Fisher test, $p<0.001$ ) (Table 2).

Other host factors, such as sex, $\mathrm{ABO}$ and Rhesus blood group, or Glucose-6-phosphate dehydrogenase deficiency, showed no influence on allele distribution. The random effects logistic regression model also allowed an investigation of the potential effect of the geographic distribution of the blood donors within the village, considered a random perturbation. This was a potential confounding factor in an analysis investigating the influence of human genetic traits. Importantly, this indicated that the location of compounds did not affect any parameter investigated.

In a separate analysis, we compared the frequency of carriage of individual alleles by group considered in the stratification, i.e., concentrating on human population and not on parasite population. This yielded essentially the same conclusions. The exact Fisher test indicated a significant imbalance of the Kl-e, Kl-h, and RO33-a allele distribution in the AS versus AA subjects $(p<0.000006$, for the first two and $p<$ 0.0036 for the last one) and for the FC27-D MSP-2 allele $(p<0.0036)$. The K1-d MSP-1 allele showed an increased frequency in the AA subjects as compared with the sickle-cell trait carriers (Khi2 $=15.1, p<0.0001)$. Likewise, there was an age-dependent imbalance of the 
TABLE 3. Distribution of individual MSP-1 alleles in the AS and AA schoolchildren recruited in Dienga, Gabon

\begin{tabular}{cccccc} 
& $\begin{array}{c}\text { No. of } \\
\text { Individuals }\end{array}$ & $\begin{array}{c}\text { No. of } \\
\text { Positive } \\
\text { Smears }\end{array}$ & $\begin{array}{c}\text { No. } \\
\text { PCR+ }\end{array}$ & $\begin{array}{c}\text { Total } \\
\text { No. of } \\
\text { Bands }\end{array}$ & $\begin{array}{c}\text { No. of } \\
\text { Bands/Carrier }\end{array}$ \\
\hline Total & 163 & 31 & 51 & 85 & 1.7 \\
AS & 40 & 9 & 12 & 21 & 1.7 \\
AA & 123 & 22 & 39 & 64 & 1.6 \\
\hline
\end{tabular}

MAD20-e and RO33-c MSP-1 allele (Khi2 $=6.27$ and 28.7, $p=0.012$ and 0.0001 , respectively).

To verify the influence of the sickle-cell trait on allele distribution, a similar genotyping strategy was used in a single age-group in another endemic area. We recruited 163 Gabonese schoolchildren aged 7-14 years, living in the endemic village of Dienga in Gabon, Central Africa (17). In this cohort, parasite prevalence and density were unaffected by age or hemoglobin type (data not shown). As shown in Table 3, 31 children had a positive blood smear and 51 were positive by MSP-1 PCR. In this village also, a very large polymorphism of the parasite population was observed. Eleven MSP-1 and 24 MSP-2 alleles were detected. The frequency of the various allelic families differed from that of Dielmo. This is not surprising, as major geographic differences in allelic frequency have been reported for several $P$. falciparum loci, including those investigated here (34). Figure 2 shows that in this cohort too, the distribution of MSP-1 families within the parasite population differed in AS and AA infected subjects (Exact Fisher test, $p<$ $0.005)$. Interestingly, the distribution of MSP-1 families observed for the parasite population infecting AS children from Dienga was very similar to that detected in AS subjects from Dielmo, particularly those with a low frequency of MAD20 alleles. As previously observed in Dielmo, the distribution of individual MSP-1 alleles was skewed by the hemoglobin type of the host (Table 3). An enhanced frequency of the $420 \mathrm{bp} \mathrm{Kl}$ allele was observed in AS as compared with AA children $(p<0.03)$. There was no difference in the frequency of the RO33 allele, nor in the frequency of MSP-2 allelic families or individual MSP-2 alleles by hemoglobin type (data not shown).

\section{DISCUSSION}

The observations reported here constitute the first indication of an imbalanced distribution of $P$. falciparum parasite genotypes related to the sickle-cell trait, a host factor conferring a strong protection against fatal and clinical malaria but not against infection. In two different areas of Africa, we have observed that the hemoglobin type strongly influences the genetic characteristics of $P$. falciparum strains hosted by asymptomatic carriers, as manifested by a lower frequency of MAD20-type alleles and enhanced frequency of specific K1-type MSP-1 alleles in parasites infecting AS as compared with AA individuals. As the parasite populations circulating in the areas in-

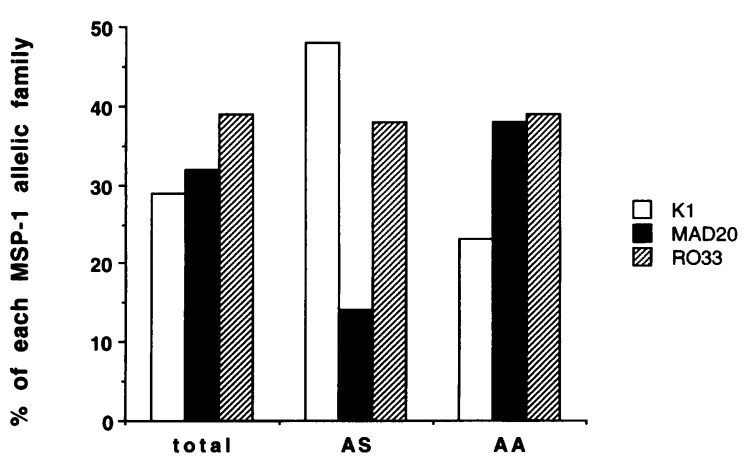

FIG. 2. Frequency of the MSP-1 allelic families in the schoolchildren recruited in Dienga, Gabon, unstratified (total) and by hemoglobin type

Ages ranged from 7 to $\leq 14$ years ( 116 year-old, 23 8-year-old, 27 9-year-old, 30 10-year-old, 2111 year-old, 22 12-year-old, 15 13-year-old and 14 14-year-old children). The mean age in the AA and in the AS group was 10.3 and 10.5 years, respectively. 
TABLE 3. Continued

\begin{tabular}{|c|c|c|c|c|c|c|c|c|c|c|}
\hline \multicolumn{5}{|c|}{ K1 } & \multicolumn{5}{|c|}{ MAD20 } & \multirow{2}{*}{$\frac{\text { RO33 }}{\text { ్ㅗ․ }}$} \\
\hline 아 & 옥 & : & $\stackrel{\circ}{m}$ & 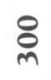 & $\underset{m}{\text { ¿ }}$ & : & 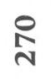 & $\stackrel{\text { ஸे }}{\text { N }}$ & ్ㅗ & \\
\hline 2 & 17 & 3 & 1 & 2 & 4 & 1 & 1 & 11 & 10 & 33 \\
\hline 0 & 8 & 1 & 0 & 1 & 0 & 0 & 0 & 1 & 2 & 8 \\
\hline 2 & 9 & 2 & 1 & 1 & 4 & 1 & 1 & 10 & 8 & 25 \\
\hline
\end{tabular}

No. of individuals refers to the number of individuals in a particular group. No. of positive smears refers to the number of samples with thick blood smears positive for P. falciparum asexual or sexual stages by microscopic examination. No. of PCR+ refers to the number of individuals who had a MSP-1-positive PCR. Total no. of bands refers to the sum of MSP-1 PCR fragments detected in all samples. No. of bands/carrier indicates the average number of distinct MSP-1 alleles detected per PCRpositive isolate. Individual MSP-1 alleles were identified as indicated in Materials and Methods. The size of the alleles (in $\mathrm{bp}$ ) is indicated. For each individual allele, the number of samples in which this allele was detected is shown. Statistically significant difference in allele frequency (calculated as number of samples in which this allele has been detected divided by total number of MSP-1 fragments) is indicated in a box.

vestigated are different, confirming the geographic parasite heterogeneity reported by others (34), this suggests that the sickle-cell trait imposes in both cases a similar constraint on the parasites.

The influence of the sickle-cell trait on the $P$. falciparum genotype concerned mainly the MSP-1 locus. The imbalance observed for a single MSP-2 allele in the Dielmo villagers was not confirmed in the Dienga cohort. The same trend in imbalanced MSP-1 allele distribution in both cohorts points to some specific association with MSP-1 or with a genetically linked marker. One possible mechanism could be reduced fitness of parasites with MAD20-type MSP-1 alleles and inversely, enhanced infectivity of parasites carrying some particular $\mathrm{Kl}$ alleles for AS cells. As the MSP-1 protein is present throughout schizont development, it is conceivable that specific interactions influenced by the MSP-1 allelic type occur at a stage where the parasite obviously interacts with the intracellular milieu. Such a scenario is compatible with the in vitro observations showing impaired maturation of FCR3 parasites (which carry a MAD20-type MSP-1 allele) in AS cells (9). Alternative mechanisms could be premature elimination of AS infected cells, due to impaired cytoadherence of some parasites when hosted in AS cells and/or increased immune clearance of certain strains carrying specific allelic forms to which AS subjects would respond more efficiently than to other allelic forms. Work is in progress in our laboratory to investigate these hypotheses.

The interpretation that $P$. falciparum strains differ in their host range is consistent with Allison's observation that only 2 of 15 AS as opposed to 14 of 15 AA Kenyan adults who had left an endemic area for 18 months and as a result had a reduced immunity, developed a blood-stage infection after experimental inoculation with the same parasite strains (15). This is also in line with our observation that $P$. falciparum strains showed substantial differences in their capacity to expand in naive Saimiri sciureus monkeys with similar genetic background (16), indicating that different parasite strains vary in their infectiousness for a particular host. Likewise, murine malaria models have shown that the infection pattern by one parasite clone differs with the mouse strain and inversely, that there is clonal parasite heterogeneity in the outcome of infection for a particular host (35). Host-dependent parasite fitness is well known for $P$. vivax, where not only some strains but the entire species is unable to infect Duffy-negative individuals (2). As a consequence, $P$. vivax is absent from most parts of Africa where this trait prevails. The preferential association of certain $P$. falciparum genotypes with hosts presenting the sickle-cell trait is predicted to reduce their reproductive rate, as prevalence of sickle-cell trait is no more than $15-20 \%$ 
in Africa $(1,36)$. Recent evidence showing that AS gametocyte carriers are more infectious to mosquitoes than AA subjects (37) indicates that increased transmissibility from AS individuals might compensate for the lower number of hosts.

Age, sickle-cell trait, and HLA Bw53 have a different impact on incidence of severe and uncomplicated malaria, and thus they are likely to protect an individual through distinct mechanisms. The observation that age but not the innate factors studied affected parasite density and complexity of the infection is consistent with the interpretation that age here reflects acquired immunity. Likewise, the specific influence of age and sickle-cell trait on allele distribution in Dielmo suggests their involvement in distinct host-parasite interactions. The reduced frequency of RO33-type alleles in the older agegroup in Dielmo is consistent with the hypothesis that acquired immunity efficiently eliminates parasites carrying the most conserved alleles. Whether this involves immune recognition of the RO33 serotype itself or of parasite factors presenting a linkage disequilibrium with the RO33 family is yet to be determined.

These data suggest that both the host's and parasite's genotype condition parasite fitness in a $P$. falciparum infection. The observations reported here are consistent with our working hypothesis of a strain-dependent host range. The possibility that sickle-cell trait might protect by reduced hospitality of AS cells for a subset of parasite genotypes opens novel clues for identification of factors contributing to virulence, a major gap in our understanding of malaria parasites. It is tempting to speculate that such host-range restriction might be involved in the protection afforded by other genetic traits. As there are marked geographical differences in parasite genotypes, the pressure exerted on host-parasite interaction may differ geographically, and this could explain why distinct protective genetic traits are observed in different endemic areas $(2,36)$.

\section{ACKNOWLEDGMENTS}

We thank the Dielmo villagers, the schoolchildren of Dienga, and their parents, who generously accepted to participate in this study. We are indebted to Gora Ndiaye, Charles Bouganali, Assane Badji, and Adrian Luty for collection of blood samples in Dielmo and Dienga, respec- tively. We also thank Badara Cisse and Adama Tall, Jean-Louis Sarthou, and Christian Roussilhon for expert assistance and Pierre Druilhe and Luiz Pereira da Silva for their invaluable input in the Dielmo program. We thank the Dienga school staff and Yakhya Dieye, Justice Mayomobo, Fautin Lekoulou, Paul Tshipamba, and Hélène Tiga for technical assistance. Particular thanks are due to Trinh Ngoe Minh for his help in the statistical analysis of the Dienga data and to André Spiegel for helpful advice in the statistical analysis of the Dielmo data. Part of this work was done when Francine Ntoumi was a fellow of the UNDP, World Bank, WHO Special Program for Research and Training in Tropical Diseases. The Dielmo work was funded by a grant from the Ministère de la Coopération. The work in Gabon was funded by grants from the Gabonese government, ELF Gabon, the Ministère de la Coopération, and INSERM. We thank Françoise Guinet, Peter David, Charlotte Behr, Florence Robert, and Jean-Louis Pérignon for critical reading of the manuscript.

\section{REFERENCES}

1. Allison AC. (1954) The distribution of the sickle-cell trait in East Africa and elsewhere, and its apparent relationship to the incidence of subtertian malaria. Trans. R. Soc. Trop. Med. Hyg. 48: 312-318.

2. Miller L. (1994) Impact of malaria on genetic polymorphism and genetic diseases in Africans and African Americans. Proc. Natl. Acad. Sci. U.S.A. 91: 2415-2419.

3. Fleming AF, Storey J, Molineaux L, Iroko EA, Attai EDE. (1979) Abnormal haemoglobins in the Sudan savanna of Nigeria. I. Prevalence of haemoglobins and relationship between sickle cell trait, malaria and survival. Ann. Trop. Med. Parasitol. 73: 161-172.

4. Hill AVS, Allsopp CEM, Kwiatkowski D, Anstey NM, Twumasi P, Rowe PA, Bennett $S$, Brewster D, McMichael AJ, Greenwood BM. (1991) Common West African HLA antigens are associated with protection from severe malaria. Nature 352: 595-600.

5. Chippaux J-P, Massougbodji A, Boulard J-C, Akogbeto M. (1992) Etude de la morbidité palustre et de la gravité des accès pernicieux chez les porteurs du trait drépanocytaire. Rev. Epidémiol. Santé Publ. 40: 240-245.

6. Chippaux J-P, Massougbodji A, Castel J, Akogbeto M, Zohoun I, Zohoun T. (1992) 
Parasitémies à Plasmodium falciparum ou $P$. malariae chez les porteurs du trait drépanocytaire dans différents biotopes du Bénin. Rev. Epidémiol. Santé Publ. 40: 246-251.

7. Bayoumi RA, Abu-Zeid YA, Abdulhadi NH, Saeed BO, Theander TG, Hviid L, Ghalid HW, Nugud HD, Jepsen S, Jensen JB. (1990) Cell-mediated immune responses to Plasmodium falciparum purified soluble antigens in sickle-cell traits subjects. Immunol. Lett. 25: 243-250.

8. Abu-Zeid YA, Abdulhadi NH, Hviid L, Theander TG, Saeed BO, Jepsen S, Bayoumi RA. (1991) Lymphoproliferative responses to Plasmodium falciparum antigens in children with and without the sickle cell trait. Scand. J. Immunol. 34: 237-242.

9. Friedman MJ. (1978) Erythrocytic mechanism of sickle cell resistance to malaria. Proc. Natl. Acad. Sci. U.S.A. 75: 1994-1997.

10. Pasvol G, Weatherall DJ, Wilson RJM. (1978) Cellular mechanism for the protective effect of haemoglobin $S$ against $P$. falciparum malaria. Nature 274: 701-703.

11. Roth EF, Friedman M, Uead Y, Tellze I, Trager W, Nagel RL. (1978) Sickling rates of human AS red cells infected in vitro with Plasmodium falciparum malaria. Science 202: 650-652.

12. Mozzarelli A, Hofrichter J, Eaton WA. (1987) Delay time of hemoglobin S polymerization prevents most cells from sickling in vivo. Science 237: 500-506.

13. Rogier C, Commenges D, Trape J-F. (1996) Evidence for an age-dependent pyrogenic threshold of Plasmodium falciparum parasitemia in highly endemic populations. Am. J. Trop. Med. Hyg. 54: 613-619.

14. Marsh K, Otoo L, Hayes RJ, Carson DC, Greenwood BM. (1989) Antibodies to blood stage antigens of Plasmodium falciparum in rural Gambians and their relation to protection against infection. Trans. $R$. Soc. Trop. Med. Hyg. 83: 293-303.

15. Allison AC. (1954) Protection afforded by sickle-cell trait against sub-tertian malarial infection. Br. Med. J. 1: 290-294.

16. Fandeur $T$, Mercereau-Puijalon $O$, Bonnemains B. (1996) Plasmodium falciparum: Genetic diversity of several strains infectious for the squirrel monkey (Saimiri sciureus). Exp. Parasitol. 84: 1-15.

17. Cooper JA. (1993) Merozoite surface antigen-1 of Plasmodium. Parasitol. Today 9: 5054.
18. Fenton B, Clark JT, Khan CMA, Robinson JV, Walliker D, Ridley R, Scaife JG, McBride JS. (1991) Structural and antigenic polymorphism of the 35- to 48-kilodalton merozoite surface antigen (MSA-2) of the malaria parasite Plasmodium falciparum. Mol. Cell. Biol. 11: 963-971.

19. Miller LH, Roberts T, Shahabuddin M, McCutchan TF. (1993) Analysis of sequence diversity in the Plasmodium falciparum merozoite surface protein-1 (MSP-1). Mol. Biochem. Parasitol. 59: 1-14.

20. Snewin VA, Herrera M, Sanchez G, Scherf A, Langsley G, Herrera S. (1991) Polymorphism of the alleles of the merozoite surface antigens MSA1 and MSA2 in Plasmodium falciparum wild isolates from Colombia. Mol. Biochem. Parasitol. 49: 265-276.

21. Prescott N, Stowers AW, Cheng Q, Bobogare A, Rzepczyk CM, Saul A. (1994) Plasmodium falciparum genetic diversity can be characterized using the polymorphic merozoite surface antigen 2 (MSA-2) gene as a single locus marker. Mol. Biochem. Parasitol. 63: 203-212.

22. Viriyakosol S, Siripon N, Petcharapirat C, Petcharapirat P, Jarra W, Thaithong S, Brown KN, Snounou G. (1995) Genotyping of Plasmodium falciparum isolates by the polymerase chain reaction and potential uses in epidemiological studies. Bull WHO 73: 8595.

23. Contamin H, Fandeur T, Bonnefoy S, Skouri F, Ntoumi F, Mercereau-Puijalon O. (1995) PCR typing of field isolates of Plasmodium falciparum. J. Clin. Microbiol. 33: 944-951.

24. Ntoumi F, Contamin H, Rogier C, Bonnefoy S, Trape J-F, Mercereau-Puijalon O. (1995) Age-dependent carriage of multiple Plasmodium falciparum merozoite surface antigen-2 alleles in asymptomatic malaria infections. Am. J. Trop. Med. Hyg. 52: 81-88.

25. Robert F, Ntoumi F, Angel G, Diatta B, Rogier C, Fandeur T, Sarthou J-L, MercereauPuijalon O. (1996) Extensive genetic diversity of Plasmodium falciparum isolates collected from patients with severe malaria in Dakar, Sénégal. Trans. R. Soc. Trop. Med. Hyg. 90: 704-711.

26. Trape J-F, Rogier C, Konate L, Diagne N, Bouganali $\mathrm{H}$, Canque B, Legros F, Badji A, Ndiaye $G$, Ndiaye $P$, Brahimi $K$, Faye $O$, Druilhe P, Pereira da Silva L. (1994) The Dielmo project: A longitudinal study of natural malaria infection and the mechanisms of protective immunity in a community liv- 
ing in a holoendemic area of Senegal. Am. J. Trop. Med. Hyg. 51: 123-137.

27. Luty AJF, Mayombo J, Lekoulou F, Mshana R. (1994) Immunologic responses to soluble exoantigens of Plasmodium falciparum in Gabonese children exposed to continuous intense infection. Am. J. Trop. Med. Hyg. 51: 720-729.

28. Dieye A, Diaw ML, Rogier C, Trape J-F, Sarthou J-L. (1996) HLA-A, -B, -C, -DR, -DQ typing in a population group of Senegal: Distribution of HLA antigens and HLADRB $1 * 13$ and DRB1*11 subtyping by PCR using sequence-specific primers (PCR-SSP). Tissue Antigens 47: 194-199.

29. O'Quigley J, Schwartz D. (1986) The comparison of several proportions in the presence of low cell expectations. Rev. Epidémiol. Santé Publ. 34: 18-22.

30. Fleiss J. (1981) Statistical Methods for Rates and Proportions. John Wiley \& Sons, New York, pp. 225-233.

31. Stiratelli R, Laird NM, Ware JH. (1984) Random-effects models for serial observations with binary response. Biometrics 40: 961-971.

Communicated by L. Miller. Accepted June 24, 1997.
32. Trape J-F, Rogier C. (1996) Combating malaria morbidity and mortality by reducing transmission. Parasitol. Today 12: 236-240.

33. Barrie Wetherill G. (1981) Intermediate Statistical Methods. Chapman and Hall, New York, pp. 254-255.

34. Creasey A, Fenton B, Walker A, Thaitong S, Oliveira S, Mutambu S, Walliker D. (1990) Genetic diversity of Plasmodium falciparum shows geographical variation. Am. J. Trop. Med. Hyg. 42: 403-413.

35. Cox FEG. (1988) Major animal models in malaria research: Rodent. In: Wernsdorfer WH McGregor I (eds). Malaria: Principles and Practice of Malariology. Churchill Livingstone, London, pp. 1503-1543.

36. Luzzatto L. (1979) Genetics of red cells and susceptibility to malaria. Blood 54: 961976.

37. Robert V, Tchuinkam T, Mulder B, Bodo J-M, Verhave J-P, Carnevale P, Nagel RL. (1996) Effect of the sickle cell trait status of gametocyte carriers of Plasmodium falciparum on infectivity to Anophelines. Am. J. Trop. Med. Hyg. 54: 111-113. 